\title{
Gyrokinetic Microinstabilities in ASDEX Upgrade Edge Plasmas
}

\author{
D. Told,${ }^{1}$ F. Jenko, ${ }^{1}$ P. Xanthopoulos,${ }^{2}$ L.D. Horton, ${ }^{1}$ E. Wolfrum, ${ }^{1}$ and ASDEX Upgrade Team \\ ${ }^{1}$ Max-Planck-Institut für Plasmaphysik, Boltzmannstr. 2, D-85748 Garching, Germany \\ ${ }^{2}$ Max-Planck-Institut für Plasmaphysik, Teilinstitut Greifswald, \\ Wendelsteinstr. 1, D-17491 Greifswald, Germany
}

\begin{abstract}
Results of linear gyrokinetic simulations of ASDEX Upgrade [O. Gruber et al, Nucl. Fusion 39, 1321 (1999)] edge plasmas, with experimentally determined geometry and input parameters, are presented. It is found that in the near-edge region, microtearing modes can exist under conditions found in conventional tokamaks. As one enters the steep-gradient region, the growth rate spectrum is dominated - down to very low wavenumbers - by electron temperature gradient (ETG) modes. The latter tend to peak near the X-point(s) and possess properties which may explain the ratios of the density and temperature gradient scale lengths that have been observed in various experiments over the last decade.
\end{abstract}

\section{INTRODUCTION}

Over the last few years, linear and nonlinear gyrokinetic simulations have emerged as a useful tool for analyzing and interpreting turbulent transport or fluctuation measurements in tokamak core plasmas (see, e.g., Ref. [1] and references therein). Meanwhile, simulations for the plasma edge have primarily been done in simplified model geometries and/or with simplified physics models. Thus, it is the main goal of the present work to identify and characterize the microinstabilities driving the turbulence in tokamak edge plasmas by means of (local) gyrokinetic simulations based on real magnetohydrodynamic equilibria for two ASDEX Upgrade [2] discharges. As it will turn out, such a more realistic description allows for new physical effects which shed some light, in particular, on the question which modes are likely to be responsible for the residual turbulent transport in the steep gradient zone of an H-mode plasma. Moreover, our results imply the necessity to reconsider the role of magnetic transport close to the edge of a tokamak.

A very good summary of the work until the mid 1980's concerning the identification and description of the relevant microinstabilites in tokamaks can be found in the review by Liewer [3]. Therein, one finds, e.g., a discussion of ion temperature gradient (ITG) modes, trapped electron modes (TEMs), trapped ion modes (TIMs), (electrostatic or electromagnetic, collisionless or collisional) electron drift waves, microtearing modes, and kinetic ballooning modes (KBMs). However, many of the early investigations referred to in this paper were based on very simple model geometries (like a sheared slab) or on simple physics models (like few-field fluid equations). Moreover, although electron drift waves had been a main candidate for driving the turbulence in edge plasmas since the pioneering theoretical work by Hasegawa and Mima [4], most of the other modes just mentioned were not associated specifically with the tokamak edge, in part due to a significant lack of realism of the underlying models.

In the course of the 1990's, the first nonlinear (Braginskii-type) fluid simulations including toroidal effects became available. Systematic studies done by Drake and co-workers [5-7] employing a circular cross section, large aspect ratio model geometry [8] indicated that one should expect, in particular, ITG modes, resistive balloning modes, and electron drift waves to dominate Lmode edge plasmas. A similar conclusion (emphasizing, however, the role of nonlinear effects) was reached independently by Scott [9] who extended his studies to gyrofluid models [10] and realistic geometry [11]. The first gyrokinetic turbulence simulations for L-mode and H-mode edge plasmas - but employing a simple model geometry - were reported in 2004 [12]. Here, besides toroidal electron drift waves, electron temperature gradient (ETG) modes were observed to play an important role in the H-mode case (when long-wavelength modes are suppressed by equilibrium $E \times B$ shear). The present work is intended to extend these previous studies to (local) gyrokinetic simulations including, in particular, trapped as well as passing particles, finite-beta effects, collisions, sub-ion-gyroradius physics, and realistic magnetohydrodynamic equilibria for two ASDEX Upgrade discharges.

The remainder of this paper is organized as follows. In Sec. II, we present some information on the experimental cases chosen for our simulations. In addition, we make some remarks about the employed simulation code and the input parameters that were used. In Sec. III, we show the results of our simulations. This section is divided into four parts, one for general core and edge results each, and one each for special phenomena found in these regions. Finally, in Sec. IV, we summarize our key results and present a brief outlook.

\section{SOME BACKGROUND ON THE EXPERIMENTS AND SIMULATIONS}

\section{A. Choice of experimental conditions}

The main goal of the present work is to perform a linear gyrokinetic analysis for ASDEX Upgrade edge plasmas with the GENE $[13,14]$ code, and to compare the properties of the resulting microinstabilities with known experimental transport characteristics in both L-mode and 
H-mode. To this aim, we will examine two discharges, namely the shots \#20431 and \#22838, which display the following features:

- Discharge \#20431 was a 1.0 MA, 2.4 T shot and had, in the time slice analysed in this paper, 5.0 MW of NBI and 2.5 MW of ICRH ( $\mathrm{H}$ minority) heating. At this heating level and with a line averaged density of $4.6 \cdot 10^{19} \mathrm{~m}^{-3}$, the input power was a factor of seven above the $\mathrm{H}$-mode threshold power and the discharge had clear Type I ELMs with a confinement enhancement factor of 1.1. Therefore, this case is chosen as an example of a discharge that is deep in the $\mathrm{H}$-mode regime.

- Discharge \#22838, on the other hand, was a 1.0 MA shot with a B-field ramp (about -2.9 T) to achieve an L-H transition, while the heating -1.5 MW of NBI - was chosen to be close to the L-H threshold. We study the L-mode phase (around $t=1.8 \mathrm{~s}$ ) as well as the H-mode phase (around $t=$ $2.8 \mathrm{~s}$ ) to gain insight into the differences between the microinstabilities in established L- and H-modes but relatively close to the $\mathrm{L}-\mathrm{H}$ transition.

As the behavior of shot \#22838 will turn out to be very similar to that of shot \#20431, we will not show and discuss the results for this discharge to the same level of detail. Instead we will concentrate on pointing out certain similarities and key differences. Unless stated otherwise, our results refer to shot \#20431.

It should also be noted that the MHD equilibrium we used for shot \#20431 has been reworked for better edge accuracy, taking into account the large pressure gradients there. Balancing these in the Grad-Shafranov equation generates a peak in the edge current profile that reinforces the poloidal field and thus causes the kink in the $q$ profile (close to the separatrix) observed in Fig. 1. The MHD equilibrium for the second discharge, \#22838, lacks this special treatment and thus also the kink in the $q$ profile. This results in considerably larger magnetic shear at the edge. As we will see, however, this difference does not lead to qualitative changes in the simulation results.

Moreover, we would like to mention that shot \#22838 displays very interesting edge transport properties. In particular, it was measured that:

- the ion heat transport is at the neoclassical level both in $\mathrm{H}$ - and L-mode phases, such that there should be no turbulence associated with significant ion heat fluxes (like ITG modes);

- the electron heat transport is anomalous, exhibiting similar transport levels in L- as in H-mode phases;

- the particle diffusivity is anomalous, showing significant differences between L- and H-mode plasmas.

These features are somewhat unconventional regarding the observed features of the L-mode phases, pointing

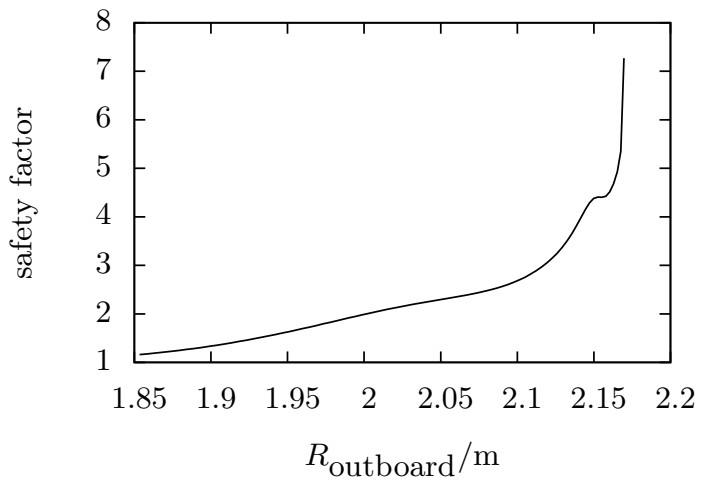

FIG. 1: Safety factor profile for shot \#20431 at $t=1.82 \mathrm{~s}$.

to the possibility that long-wavelength microinstabilities (and turbulence) are suppressed by equilibrium $E \times B$ shear in both regimes. Under these circumstances, turbulence at moderate to high wavenumber would determine the anomalous transport in the particle and electron heat channels. We will return to this important point after we have presented the simulation results.

\section{B. Gyrokinetic simulation details}

All simulation results shown in the present paper have been obtained by means of the gyrokinetic turbulence code GENE [13, 14] in its linear mode of operation, taking into account two particle species (electrons and deuterium ions), passing and trapped particles, electromagnetic effects, collisions (including both pitch angle and energy scattering between like and unlike particles), sub-ion-gyroradius physics, and realistic MHD equilibria. Thus, our model may be considered rather comprehensive within the limits of a local approach. Although the GENE code can track subdominant instabilities when used as an eigenvalue solver, here we only search for the most unstable modes and thus operate the code as an initial value solver. Collisions are modeled in GENE using the velocity space part of a linearized Landau-Boltzmann operator, see $[15,16]$.

The simulations are carried out with $32 \times 16$ grid points in $\left(v_{\|}, \mu\right)$ velocity space, using - depending on the complexity of the geometry under consideration - between 32 and 64 grid points in the parallel direction and typically $12 k_{x}$ values (allowing for parallel connections across the box boundary), probing only a single $k_{y}$ mode at a time. Here, the perpendicular box size is determined by the expressions $L_{y}=2 \pi / k_{y}$ and $L_{x}=1 /\left(\hat{s} k_{y}\right)$ where $\hat{s}$ is the (global) magnetic shear of the flux surface under consideration. The convergence of the results under these conditions is checked by means of careful resolution tests.

GENE employs field-aligned coordinates, and the required metric coefficients are obtained from MHD equilibria via a field-line tracing procedure described in Ref. [17]. Thus, there are no approximations or special 
assumptions being made in order to simplify or model the geometric input data. Instead, we work with the full geometric information contained in the experimental MHD files. This allows us, in particular, to perform very accurate simulations in the neighborhood of the separatrix, where the influence of the X-point is clearly reflected in the behavior of the metric coefficients.

For the simulations of shot \#20431, we choose four flux surfaces to represent distinct regions in the plasma. Fig. 2 shows the profiles we use for our edge simulations. These are obtained through hyperbolic tangent fits [18] to the data from various plasma diagnostics, averaged over the time window $t=1.65 \mathrm{~s}-2.0 \mathrm{~s}$. Table I shows our nominal input parameters, with the gradients being defined according to the formula

$$
\omega_{x}=-\frac{1}{x} \frac{d x}{d \varrho_{\text {tor }}}
$$

Here, $x$ represents the temperatures $T_{e}, T_{i}$, or the particle density $n$, while $\varrho_{\text {tor }}=\sqrt{\Phi / \Phi_{\text {edge }}}$ and $\Phi$ is the toroidal flux. For labeling the flux surfaces in this document, we use $\varrho_{\text {pol }}=\sqrt{\Psi / \Psi_{\text {edge }}}$ with the poloidal flux $\Psi$. All frequencies are given in units of $c_{s} / L_{\perp}$, where $c_{s}$ is the ion sound speed and $L_{\perp}=\sqrt{\Phi_{\text {edge }} / \pi B_{0}} ; B_{0}$ is the magnetic field at the magnetic axis. Using this definition, which is roughly the minor radius, we get $L_{\perp}=0.673 \mathrm{~m}$ for shot $\# 20431, L_{\perp}=0.672 \mathrm{~m}$ and $L_{\perp}=0.679 \mathrm{~m}$ for the L- and H-mode phases of shot \#22838, respectively.

In the case of shot \#20431, we note that the experimental uncertainties for the $T_{i}$ profile are larger than those for the $T_{e}$ and $n$ profiles. This is due to the fact that there was no edge ion temperature diagnostics available. Thus, there are only few data points to determine the fit we use. For the electron temperature and density, the measurements were taken prior to an ELM, whereas for the ion temperature there is no ELM synchronizing. The data for shot \#22838, on the other hand, included measurements from a new edge CXRS diagnostic [19] which yields a much more accurate $T_{i}$ profile.

A note of caution has to be sounded regarding the validity of gyrokinetic theory as one approaches the separatrix. There, the profile scale length (usually that of the electron temperature is the smallest) can reach values close to the ion gyroradius, which pushes the ordering imposed on gyrokinetic theory, $\epsilon=\varrho / L \ll 1$, to its very limits. The ordering parameter $\epsilon$ is particularly large at the outboard side of each flux surface, due to the lower magnetic field and the Shafranov shift, which decreases the distance between neighboring flux surfaces there. The following 'worst case' values are obtained for our flux surfaces:

\begin{tabular}{lllll}
\hline \hline$\varrho_{\text {pol }}$ & 0.5 & 0.93 & 0.96 & 0.98 \\
\hline$\epsilon=\varrho / L$ & 0.015 & 0.019 & 0.092 & 0.220 \\
\hline \hline
\end{tabular}

In the edge flux surface, this value decreases by a factor of five towards the inboard side.

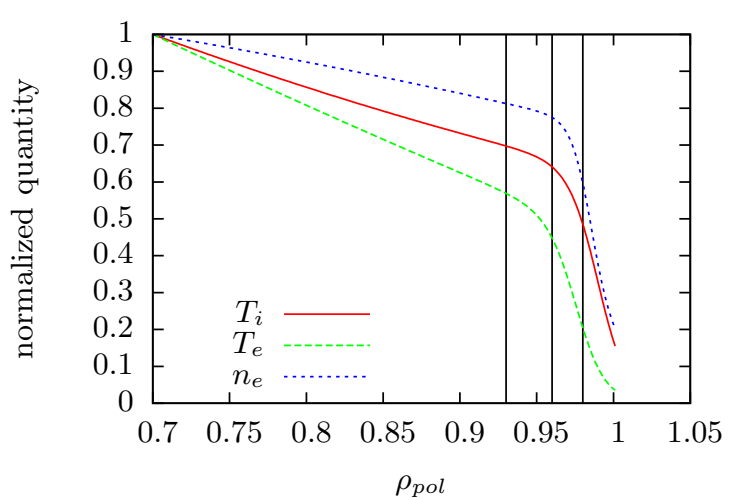

FIG. 2: (Color online) Temperature and density profiles for shot \#20431 at $t=1.82 \mathrm{~s}$. The vertical lines indicate the positions of three of the flux surfaces we simulated. As our focus is on the edge region, the fourth flux surface $\left(\varrho_{\mathrm{pol}}=0.5\right)$ is excluded from this figure.

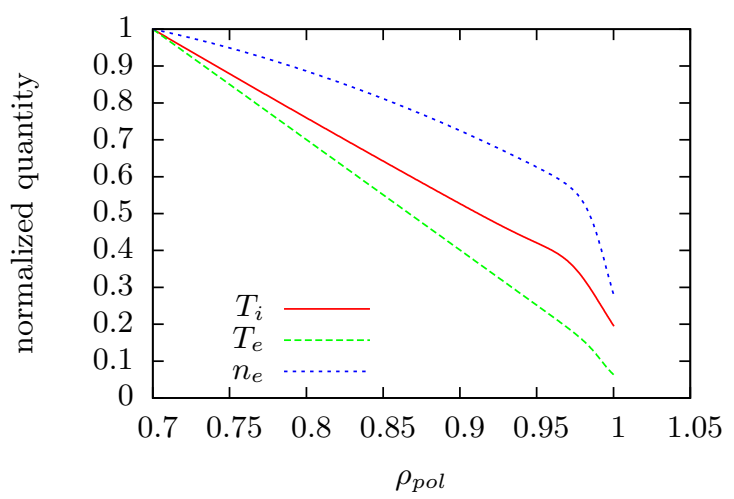

FIG. 3: (Color online) Temperature and density profiles for the L-mode phase of shot \#22838.

Furthermore, in the edge one may expect neutral particles - which are not included in gyrokinetic theory - to influence the plasma microinstabilities through collisions with the charged particles. While the electron-neutral collision frequency is comparable to the electron-ion collision frequency, we will see that the instabilities found in the edge are essentially collisionless. We thus assume the influence of neutral particles on our calculations to be small.

\section{SIMULATION RESULTS}

\section{A. Outer core plasma}

Before we focus on the main topic of the present paper, namely the identity and character of the dominant linear microinstabilities in the edge region of ASDEX Upgrade, we would first like to investigate the situation in the outer core plasma. The corresponding results are meant to serve, among other things, as a point of reference for what 


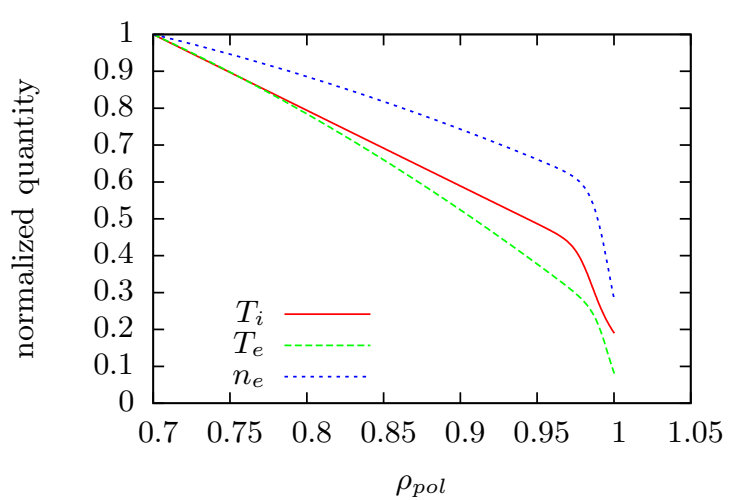

FIG. 4: (Color online) Temperature and density profiles for the H-mode phase of shot \#22838.

\begin{tabular}{lrrrrr}
\hline \hline Quantity $\backslash \varrho_{\text {pol }}$ & 0.50 & 0.93 & 0.96 & 0.98 \\
\hline$\omega_{T i}$ & 1.57 & 1.04 & 3.12 & 14.4 \\
$\omega_{T e}$ & 1.70 & 2.18 & 10.1 & 33.3 \\
$\omega_{n}$ & 0.50 & 0.69 & 1.82 & 17.0 \\
$T_{e} / \mathrm{keV}$ & 3.11 & 1.26 & 0.97 & 0.45 \\
$n_{e} / 10^{19} \mathrm{~m}^{-3}$ & 5.17 & 3.76 & 3.57 & 2.74 \\
$T_{i} / T_{e}$ & 1.19 & 1.53 & 1.81 & 2.99 \\
$\beta_{e} / \%$ & 1.26 & 0.37 & 0.27 & 0.10 \\
$\nu_{e i} / \mathrm{kHz}$ & 106 & 285 & 394 & 918 \\
$c_{s} /\left(10^{5} \mathrm{~m} / \mathrm{s}\right)$ & 3.85 & 2.46 & 2.15 & 1.47 \\
$q$ & 1.54 & 3.50 & 4.37 & 4.48 \\
$\hat{s}$ & 0.70 & 3.42 & 1.44 & 2.14 \\
\hline \hline
\end{tabular}

TABLE I: Plasma parameters for four specific flux surfaces of shot \#20431.

follows later. Thus, we will be able to better describe the differences between core and edge cases.

Indeed, for the flux surface at $\varrho_{\text {pol }}=0.5$, we obtain the same kind of microinstabilities which are commonly held responsible for core transport, based on earlier simulation work in simple $\hat{s}-\alpha$ and Miller-type geometries (see, e.g., Ref. [1]). The corresponding growth rate and frequency spectra can be found in Fig. 5. Descending from large perpendicular space scales towards smaller ones, the spectra show an ion temperature gradient (ITG) mode, spanning the $k_{y}$ range $0.04<k_{y} \varrho_{s}<0.9$, which is immediately followed by an ETG mode that persists to very large wavenumbers around 60 .

According to this finding, one may actually expect multiscale effects to be important in respective nonlinear simulations involving ITG and ETG modes at the same time. Such phenomena have indeed been observed recently (see, e.g., Refs. [20, 21] and references therein). Thus, assuming that the ITG-induced heat fluxes are not too large (i.e., the ion temperature gradient is not too far above the linear or nonlinear threshold), contributions from ETG turbulence can be expected to play a non-negligible role.

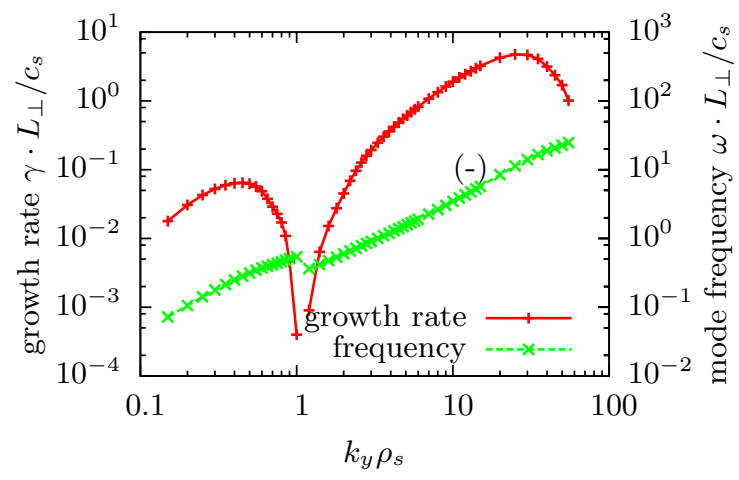

FIG. 5: (Color online) Growth rate and frequency spectra at $\varrho_{\text {pol }}=0.50$. Negative frequencies (corresponding to the electron drift direction) are indicated by '(-)'.

In the present case, the linear ITG threshold is found to be $\omega_{T i \text {,crit }} \approx 0.87$. This means that the nominal gradient is about $80 \%$ above the critical one. A simple nonlinear GENE simulation - using adiabatic electrons - for real geometry showed that the resulting ion heat fluxes are unrealistically large, as could be expected. The experimental uncertainty of the ion temperature profile is not sufficient to explain this discrepancy. However, there are other possibilities to reconcile experiment and simulation. First of all, there are no motional Stark effect (MSE) measurements available to constrain the safety factor profile in the core of shot \#20431. Gyrokinetic simulations [22] have shown, however, that the critical gradient for adiabatic ETG modes is proportional to $(1.33+1.91 \hat{s} / q)$. The dependence of the threshold on $\hat{s} / q$ carries over to ITG modes with comprehensive physics as a test with a different equilibrium indicates. As a consequence, the experimental error bars in the safety factor profile (without MSE) allow for variations of the order of $50 \%$ in the critical gradient. In addition to this, we do not take into account possible $E \times B$ shearing effects which reduce the linear growth rates of ITG modes as well as the resulting transport. For the critical gradient of the ETG mode, we find a value of $\omega_{T e \text {,crit }}=0.95$, i.e., the experimental gradient is $\sim 80 \%$ above the linear threshold. This causes less concern than in the ITG case, however, since the flux-gradient relationship of ETG transport is known to be much less stiff.

According to our findings up to this point, we are well within the boundaries of 'conventional wisdom', but interestingly, as we proceed towards the edge, the situation changes earlier than expected.

As our second core case, we show the results for $\varrho_{\text {pol }}=0.93$, which corresponds to the pedestal top. As can be seen in Figure 6, the mode frequency exhibits a sudden change of sign at $k_{y} \varrho_{s} \approx 0.5$. The lower $k_{y}$ modes rotate in the electron drift direction (negative frequency), the higher $k_{y}$ modes in the ion drift direction. An examination of the parallel mode structure reveals that the instability with negative frequency is a microtearing mode. 


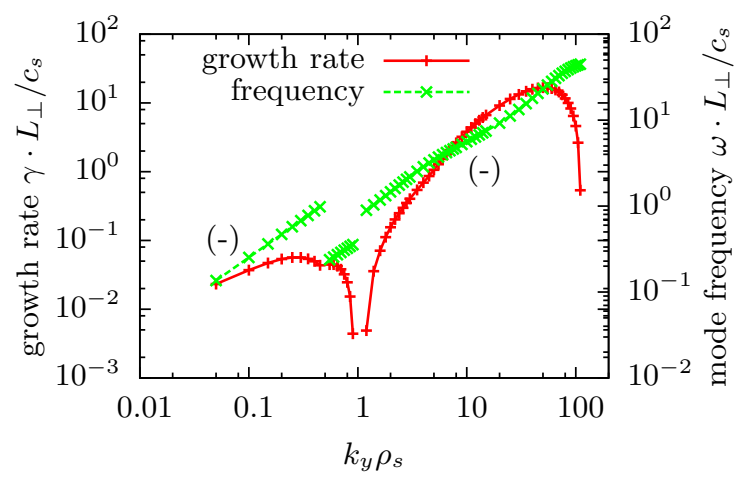

FIG. 6: (Color online) Growth rate and frequency spectra at $\varrho_{\text {pol }}=0.93$. Negative frequencies are indicated by '(-)'.

So far, this kind of plasma instability has been observed (in gyrokinetic simulations) mainly for spherical tokamak experiments, like in Ref. [24]. The study in [24] also indicated that microtearing should be found even in conventional tokamaks as it is only gradually stabilized when increasing the aspect ratio. In addition, the local aspect ratio of flux surfaces near the edge of ASDEX Upgrade is similar $(A \sim 3)$ to that of the mid-radius flux surfaces that were examined in the spherical tokamak studies. Furthermore, a recent gyrokinetic study employing a Miller-equilibrium approach also found microtearing unstable in ASDEX Upgrade H-mode conditions [23].

From a theoretical point of view, while there have been many investigations on microtearing modes since the 1970 's, a clear physical picture is still lacking - and this makes it difficult to predict when microtearing modes should be expected to be important. At present, the best strategy is to perform comprehensive gyrokinetic simulations for specific experimental cases, as presented in [24] and is done here for ASDEX Upgrade edge conditions. A more detailed study of the properties of these microtearing modes will be presented below.

The high-wavenumber region is, as in the other core case, dominated by an ETG mode. The critical gradients are $\omega_{T i \text {, crit }}=0.85$ and $\omega_{T e, \text { crit }}=0.82$, so that the experimentally determined gradients are $22 \%$ and $166 \%$ above the critical ones for the ITG and ETG modes, respectively. In Fig. 7 we show the $k_{y}$ spectrum as it appears with the ion temperature gradient set to zero (we will return to this setup in the next section), where the microtearing mode dominates up to $k_{y}=0.65$ and the ITG mode is found to be replaced by a TEM which smoothly transitions into an ETG mode towards smaller scales.

In shot \#22838, again microtearing exists in the pedestal top of both L- and H-mode phases, even though in a less pronounced way than in the other discharge. Here, when decreasing the ion temperature gradient, we find that after only a small reduction, TEM starts to dominate, making it difficult to find the critical gradient for the ITG mode. If one extrapolates the growth rate curve, one finds that the experimentally determined gra-

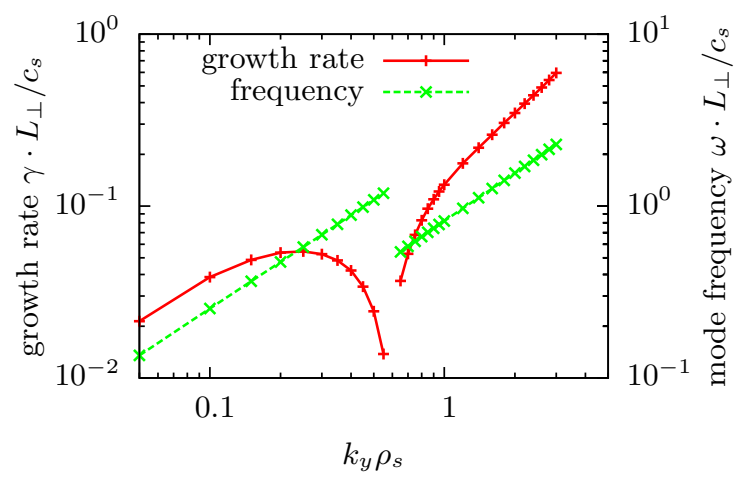

FIG. 7: (Color online) Growth rate and frequency spectra at $\varrho_{\text {pol }}=0.93$, with zero ion temperature gradient. The displayed frequencies are all negative.

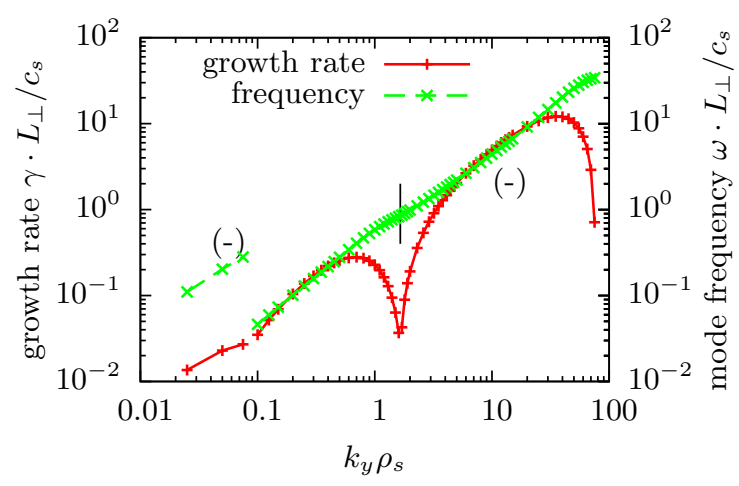

FIG. 8: (Color online) Growth rate and frequency spectra at $\varrho_{\text {pol }}=0.77$ in the L-Mode phase of shot \#22838. Negative frequencies are indicated by '(-)', while the black bar separates the ITG and ETG mode frequencies, which have similar values but a different sign.

dient is again about 50 to $80 \%$ above the linear threshold. At higher wavenumbers we find, as before, unstable ETG modes.

Further simulations show that in both discharges, microtearing also exists deeper in the core of the plasma, at values of $\varrho_{\text {pol }}$ of about 0.7 . As an example for this, we show the $k_{y}$ spectra for $\varrho_{\text {pol }}=0.77$ of the L-Mode phase, Fig. 8. One finds the familiar picture consisting of ITG and ETG modes, and a microtearing mode at the largest scales.

In summary, the outer core plasmas of the ASDEX Upgrade discharges under investigation here exhibit the usual types of microinstabilities, namely ITG modes, TEMs, and ETG modes. However, in addition to that, microtearing modes are linearly unstable in the radial region $\varrho_{\text {pol }} \sim 0.7-0.95$, i.e., just inside the pedestal top. Even at $\varrho_{\text {pol }}=0.5$, they turn out to be only marginally stable. Given its prevalent appearance under common ASDEX Upgrade conditions, the microtearing instability deserves a more detailed study which is the topic of 
the next subsection.

\section{B. Microtearing modes in the outer core plasma}

In order to better understand the nature of the microtearing modes appearing in our simulations, we now perform variations of several plasma parameters about their nominal values to assess their respective influence. A similar study has been performed in [24] for conditions as found in the MAST spherical tokamak.

For these examinations, we use the $\varrho_{\text {pol }}=0.93$ flux surface, taking the ion temperature gradient to be zero in order to avoid a competition between microtearing and ITG modes. This approximation is justified by the fact that the microtearing mode is more or less independent of the ion temperature gradient, as is shown in Fig. 9. All simulations are performed using $k_{y} \varrho_{s}=0.25$, the wavenumber corresponding to the largest microtearing mode growth rate. We employ $24 \times 32 \times 32 \times 16$ points in the radial, parallel, $v_{\|}$, and $\mu$ directions, respectively. The radial resolution has been doubled compared to before due to the very long extension of the microtearing mode along the field line.

Let us now turn to systematic variations of some of the the main plasma parameters around their experimentally determined values. First, according to Fig. 10, the mode exhibits a critical electron temperature gradient of about $\omega_{T e, \text { crit }}=1.34$. The existence of such a threshold is in line with analytical predictions [25]. The nominal value exceeds this threshold by $\sim 63 \%$. On the other hand, as we have seen above, there is virtually no dependence on the ion temperature gradient, and, in quasilinear estimates, the mode turns out not to produce large ion heat (and particle) fluxes. When varying the density gradient, one finds that the microtearing instability can also be driven by this parameter. Using our nominal pedestal top parameters, we indeed find a critical density gradient (see Fig. 11), while further inside the core we do not. There, simulations reveal that there exists a preferred value of the density gradient. In both cases, however, when the density gradient is further increased, the microtearing mode is dominated by a TEM. Our findings support those in [26], where an existing nonlinear theory was found to agree better with experimentally found transport values when the microtearing mode was assumed to be driven both by electron temperature gradient and density gradient.

Given its inherently electromagnetic nature, the microtearing mode is also expected to exhibit a critical beta value. And indeed, one finds that the mode becomes unstable only if $\beta_{e}$ exceeds a value of about $0.2 \%$, see Fig. 12. The experimental value is about twice that high. Quasilinear results regarding the different contributions to the total cross-field transport induced by these modes show that the dominant transport channel is the magnetic electron heat transport, confirming the electromagnetic character of the instability. One more signature of this mode, related to the feature just described, is the even (odd) parity of its $A_{\|}(\phi)$ component with respect to the outboard midplane position (where it generally peaks, see Fig. 13 and, e.g., Ref. [27]).

Finally, several theoretical studies since the 1970's (see, e.g., Refs. [25, 28, 29]) pointed out that the presence of collisions greatly facilitates the destabilization of microtearing modes. Thus, one generally expects that such microinstabilities are more important in (radial regions of) discharges with a relatively high collisionality value, e.g., relatively close to the edge of a tokamak. In our present case, a collisionality scan (see Fig. 14) reveals that there indeed exists a threshold value - which is clearly exceeded here, however. In the collisionless limit, the growth rate of the microtearing drops substantially, and a TEM starts to dominate. Similarly as in [24], the mode is stabilized when going to high collisionalities, but here this occurs only for collisionalities greater than about $40 c_{s} / L_{\perp}$, which is about fifty times the experimental value.

In summary, we have confirmed several expectations from previous analytical studies [25, 28, 29]. Furthermore, our results agree well with recent gyrokinetic simulations of spherical tokamak plasmas [24], where similar dependencies were found. Our simulations (confirming hints from [24]) show that microtearing modes are quite a common appearance even in conventional tokamaks, which implies that the well-established picture of ITG, TE and ETG modes as main actors in tokamak microturbulence has to be extended to include the microtearing instability.

To gain a comprehensive picture and to learn whether microtearing modes can contribute significant transport, nonlinear simulations of this mode are also necessary. To our knowledge, there exists no publication to date containing such results. Nonlinear simulations of the microtearing instability are computationally expensive, as a very fine radial resolution is needed, while having large box sizes as a consequence of the low wavenumbers. In addition, the growth rates of microtearing in ASDEX Upgrade are quite small, resulting in long simulation times. We are currently running first nonlinear simulations, results of which can be expected to appear in a future publication.

\section{Plasma edge}

Moving further out into the plasma edge, a key question is how the microinstabilities react to the dramatic decrease of the gradient scale lengths. In other words, what is going to change when one is entering the steep gradient zone where normalized gradients can be larger by a factor of 20 or so with respect to the pedestal top values (see Table I)? In addition to that, the magnetic geometry is increasingly influenced by the proximity of the separatrix. In the following, we show the results for two flux surfaces, namely $\varrho_{\text {pol }}=0.96$ and $\varrho_{\text {pol }}=0.98$, 


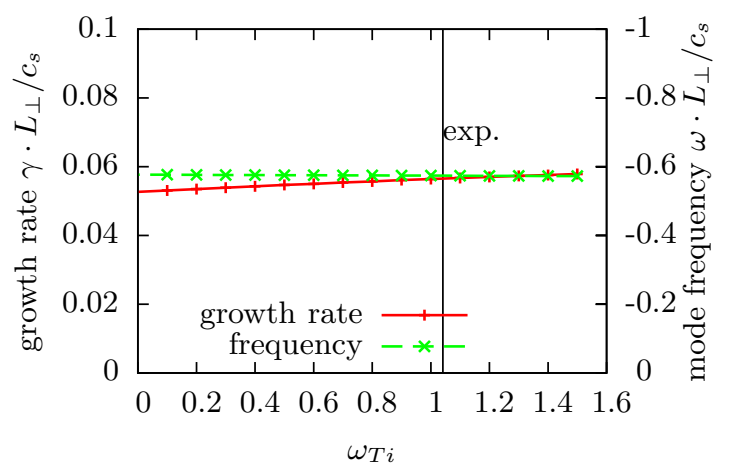

FIG. 9: (Color online) Dependence of the microtearing mode on the ion temperature gradient.

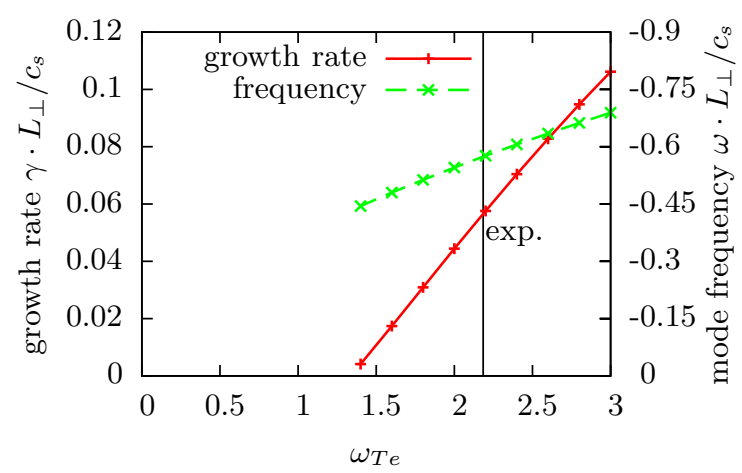

FIG. 10: (Color online) Dependence of the microtearing mode on the electron temperature gradient.

which represent, in \#20431, the pedestal shoulder (with intermediate gradients) and the steep gradient zone, respectively. There, the ordering parameter $\rho / L$, which in gyrokinetic theory is taken to be much smaller than one, reaches values as high as 0.2 in the case of ions. Thus one has to be careful when interpreting ITG results in that region.

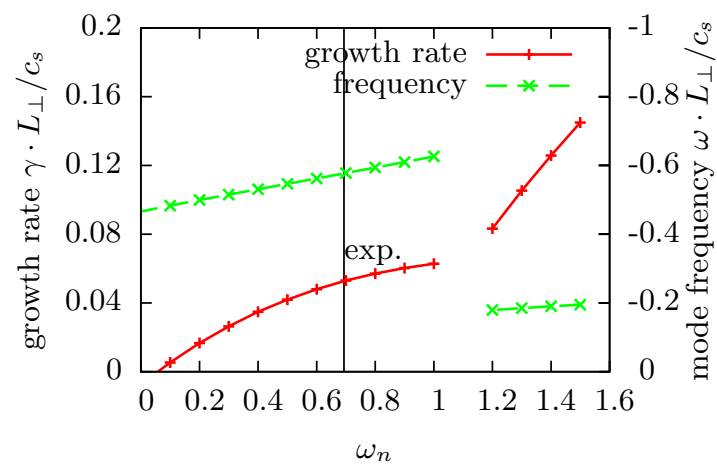

FIG. 11: (Color online) Dependence of the microtearing mode on the density gradient. At high gradient, a TEM dominates.

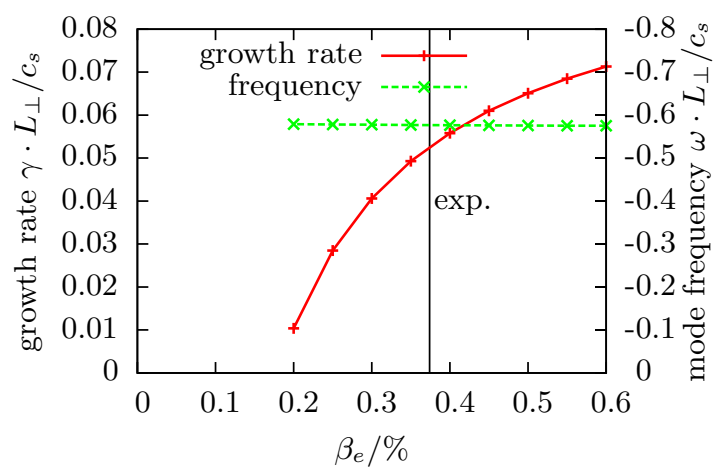

FIG. 12: (Color online) Dependence of the microtearing mode on $\beta_{e}$.

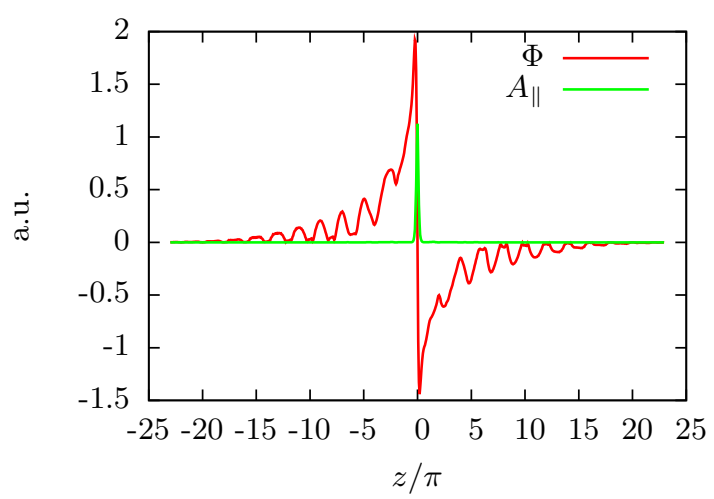

FIG. 13: (Color online) Parallel mode structure of the microtearing instability. The electrostatic potential $\Phi$ shows an odd parity, while the electromagnetic potential $A_{\|}$is of even parity.

In line with our general expectations, the character of the instabilities shows a significant change at the pedestal shoulder. The low- $k_{y}$ part of the growth rate spectrum is dominated by a mode with positive frequency (corresponding to the ion drift direction), which would normally be classified as an ITG mode. However, an ion temperature gradient scan reveals that the mode can exist even with zero temperature gradient (see Fig. 16), and that its mode frequency shows a smooth transition from positive (ion drift direction) to negative (electron drift direction) values. The instability thus shows properties both of ITG and TE modes and is very similar to the 'ubiquitous mode', which has been studied before both analytically [30] and in gyrokinetic simulations [31]. However, a recent study by Kammerer and co-workers on so-called Exceptional Points has shown that microinstabilities can be transformed into one another by smooth variations of the plasma parameters, making a unique labelling of instabilities impossible except in simple limiting cases. It is thus no surprise that hybrid modes are encountered in the pedestal shoulder, were a small variation in radial position can lead to a significant change 


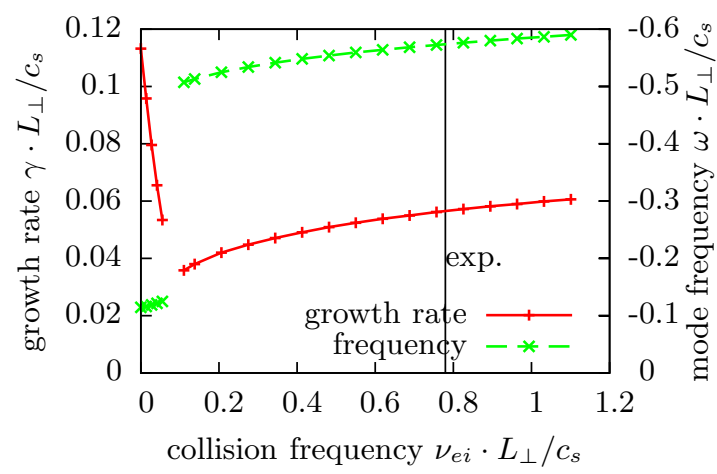

FIG. 14: (Color online) Dependence of the microtearing mode on collisionality. At low collisionality, a TEM dominates.

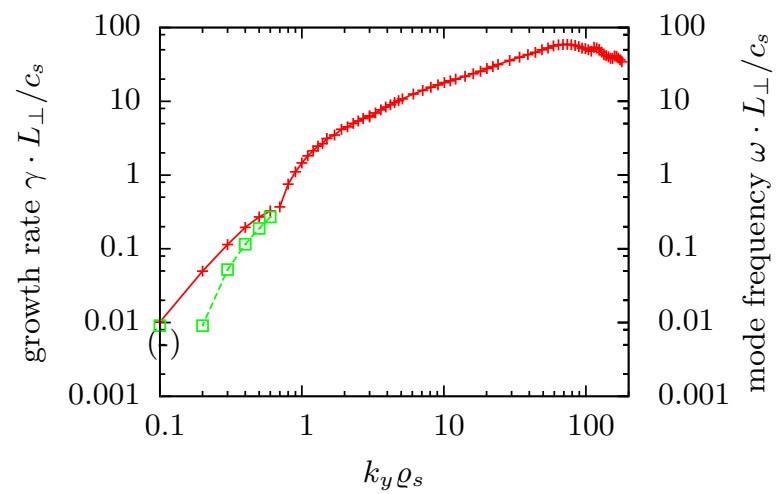

FIG. 15: (Color online) Growth rate and frequency spectra at $\varrho_{\mathrm{pol}}=0.96$. Negative frequencies are indicated by '(-)'.

in the gradients.

In the pedestal shoulder of shot \#22838, we find, both in L- and H-mode, that ITG modes dominate the largescale region. Interestingly, the conditions in the pedestal shoulder seem to inhibit microtearing, as we have not observed them in any simulations there.

In all the aforementioned cases, at higher wavenumbers one observes a different instability which happens to be an ETG mode, but with a highly unusual character. This is why we will dedicate a separate section, Sec. III D, to this mode and some of its key properties.

First, however, we would like to present some results for the large-scale regime of the steep gradient zone in shot \#20431, where we find an ITG-like mode that spans a $k_{y} \varrho_{s}$ range from $\sim 0.01$ to $\sim 0.08$ (see Fig. 17). It coexists with a microtearing mode that lies between $0.10 \lesssim k_{y} \varrho_{s} \lesssim 0.18$. In shot \#22838, on the other hand, the low- $k_{y}$ region in the steep gradient zone displays a TEM-like mode (both in L- and H-mode phases) similar to the one observed in the $\varrho_{\text {pol }}=0.96$ case of shot \#20431, but which does not depend on the ion temperature gradient and still moves in the ion drift direction.

However, the importance of such large-scale modes in the steep gradient zone is difficult to assess from our sim-

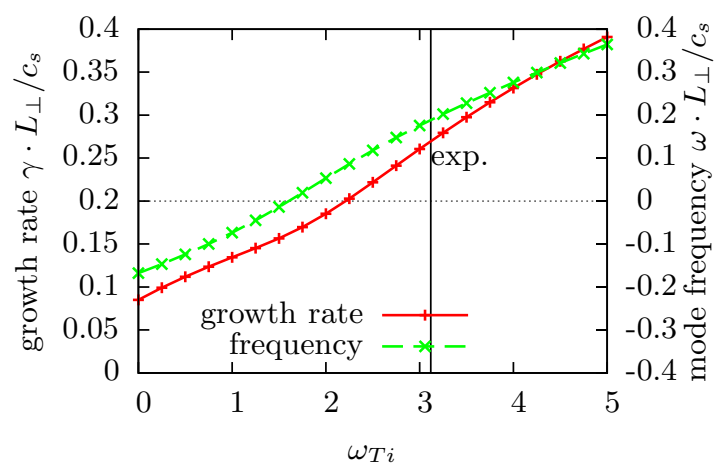

FIG. 16: (Color online) Dependence of the low- $k_{y}$ mode $\left(\varrho_{\text {pol }}=0.96\right)$ on the ion temperature gradient. The dotted line separates the ion drift direction (upper half) from the electron drift direction (lower half).

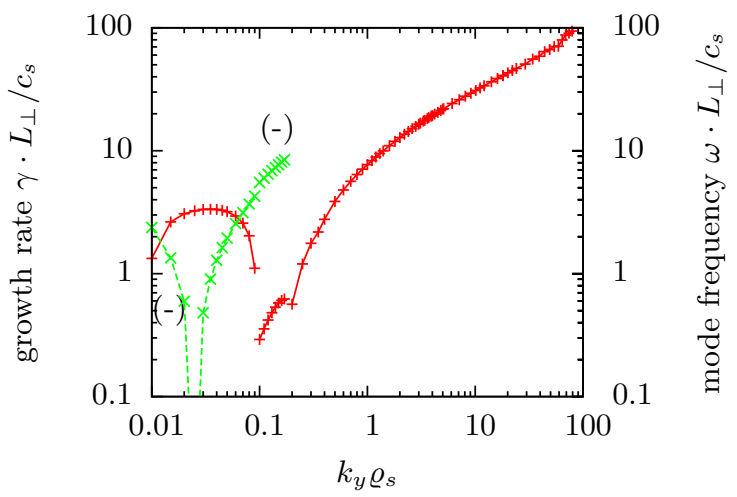

FIG. 17: (Color online) Growth rate and frequency spectra at $\varrho_{\text {pol }}=0.98$. Negative frequencies are indicated by '(-)'.

ulations: As mentioned before, the gyrokinetic ordering for ions is only marginally satisfied in the edge. In addition, the radial extension of the steep gradient zone is only about $10 \varrho_{s}$. For $k_{y} \lesssim 0.5$, the radial extension of our simulation domain (as given by the formula in Section II B) becomes comparable that of the steep gradient zone. Under such conditions, the local approximation (background plasma parameters are taken to be constant over the simulation box) used in our code breaks down. This issue is likely to be resolved by $E \times B$ shearing which is particularly strong in the plasma edge and large enough to (at least partly) decorrelate the large-scale instabilities. This effect is not taken into account in our present simulations.

\section{ETG modes in the plasma edge}

At $\varrho_{\text {pol }}=0.96$, one observes a mode at high wavenumbers $\left(k_{y} \varrho_{s} \gtrsim 0.7\right.$, see Fig. 15$)$ with unique properties. As this same mode plays an even more prominent role in the steep gradient zone (at $\varrho_{\text {pol }}=0.98$ ), where it is the most 


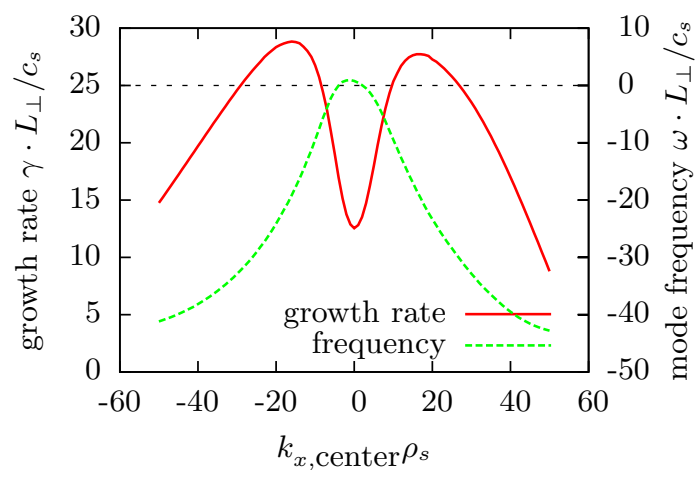

FIG. 18: (Color online) Growth rate and frequency for $k_{y} \varrho_{s}=$ 20 and variable $k_{x, \text { center }}\left(\right.$ at $\varrho_{\text {pol }}=0.96$ ); the black line separates positive from negative frequencies. The asymmetry with respect to $k_{x, \text { center }}=0$ is only possible in flux surface geometries that are not up-down symmetric.

unstable mode over almost the entire $k_{y}$ spectrum, we will now investigate its features in more detail.

Normally, linear microinstabilities peak at $k_{x, \text { center }}=$ 0 , which means that in the outboard midplane of the tokamak (i.e., at the "center" of the parallel extension of the simulation volume), the wavelength in the (radial) $x$ direction is zero. Since $k_{y}$ is always finite, this corresponds to structures which are highly elongated in the radial direction, so-called streamers.[13, 33] However, as one moves along the field line, the angle of these streamers is tilted away from the radial direction by magnetic shear. In the present case, however, to get maximal linear growth rates, one has to choose a finite $k_{x, \text { center value }}$ (Fig. 18), which means that the streamers are tilted on the outboard side, not horizontal. Further examinations show that the mode can have streamers tilted in both directions, which lets the electrostatic potential peak at the top or at the bottom (i.e., near the X-points) of the plasma, depending on the sign of $k_{x, \text { center }}$. Here, negative $k_{x, \text { center }}$ corresponds to peaking at the bottom (see Fig. 19) and vice versa.

A complication that ensues when simulating this mode

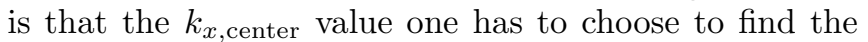
maximal growth rate is a function of $k_{y}$, forcing one to do two-dimensional scans in the $k_{x}-k_{y}$ plane to obtain a diagram like in Fig. 17. We circumvent this problem by using a script which evaluates, for each $k_{y}$ mode, at least three runs of GENE with different $k_{x \text {,center values, }}$ then estimating the maximal growth rates and the val-

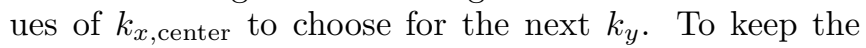
script simple, we use only one $k_{x}$ mode per run (i.e., the mode amplitude is cut off at the ends of the flux tube). This is done because the GENE code calculates only the largest growth rate within the simulation domain. Thus, other $k_{x}$ modes that are included to extend the flux tube in parallel direction (through the boundary condition described in Sec. II B) might mask the growth rate of the

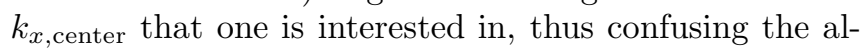

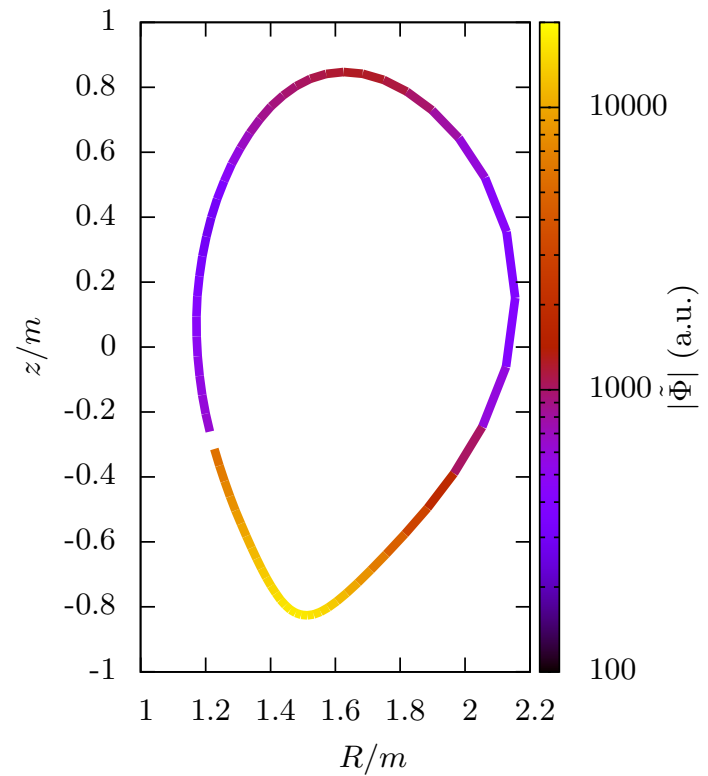

FIG. 19: (Color online) Fluctuation amplitude of the electrostatic potential, mapped to the flux surface $\varrho_{\text {pol }}=0.98$. The

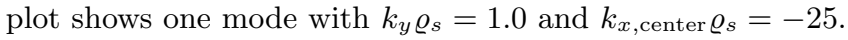

gorithm of the script.

The restriction to one $k_{x}$ mode is only possible thanks to the strongly localized parallel structure of the instability, and convergence tests have shown that there are no qualitative changes to the results shown in Figs. 15 and 17. However, we do not show frequencies for these modes. The reason for this can be seen in Fig. 18: At the $k_{x}$ of maximum growth rate, the frequency depends roughly linearly on $k_{x}$. Thus, even when the maximum growth rate is determined relatively accurately, the frequency still has a large error bar - this is not due to bad convergence of the simulation, but due to an insufficiently accurate determination of the $k_{x}$ of maximum growth rate. The importance of the frequency that the instability has exactly at the $k_{x}$ of maximal growth rate is to be questioned, as the neighboring $k_{x}$ modes have similar growth rates, but different frequencies, which will all coexist in a nonlinear simulation.

Although the instability studied here exhibits a rather unusual parallel mode structure (it does not peak at the outboard side), we label this an ETG mode. This is justified by the following properties:

- the mode exhibits a linear instability threshold of $\eta_{e}=L_{n} / L_{T} \approx 1.1-1.25$;

- the electrostatic electron heat transport is the dominant transport channel;

- collisions have practically no influence on the growth rates;

- ions can be treated adiabatically without seriously affecting the instability. 
The surprisingly large perpendicular scales to which this mode extends can be attributed to the steep gradients present at the edge. In Ref. [34], it has been shown that (within the framework of an adiabatic ion, simple model geometry treatment) ETG modes have their low$k_{y}$ cut-off at $k_{y} \rho_{e} \approx \sqrt{T_{e} / T_{i}}\left(L_{T_{e}} / q R\right)$. Thus, for typical core parameters $\left(R / L_{T_{e}} \sim 8, q \sim 1.5\right)$, one expects this cut-off to lie at $k_{y} \rho_{e} \lesssim 0.1$, while for edge parameters $\left(R / L_{T_{e}} \sim 100, q \sim 4\right)$, this formula predicts a cut-off at $k_{y} \rho_{e} \approx 0.0025$ or $k_{y} \varrho_{s} \approx 0.15$ (for a deuterium plasma). This means that an ETG mode may extend down to the typical ITG/TEM scales in the steep gradient edge region. This is exactly what is observed in the present case.

The transport generated by this edge-ETG mode shows another interesting characteristic. The instability can produce both outward and inward particle transport, depending on the value of $\eta_{i}$. For $\eta_{i} \lesssim 1$, the particle transport points outward, while for $\eta_{i} \gtrsim 1$ there is inward transport (a "particle pinch"). Note that this reversal is brought about by varying a gradient which is not the primary drive of the instability. This behavior is also found with TEMs, and may lead to the following mechanism. Consider a situation where $\eta_{i}<1$, i.e., the normalized ion temperature gradient is smaller than the normalized density gradient. The instability then generates outward particle flux. This flattens the density profile, which in turn increases $\eta_{i}$. This continues until the point is reached when the instability starts to generate a particle pinch. The inverse scenario applies when starting from $\eta_{i}>1$. This leads us to conclude that in situations where this edge-ETG mode is the dominant source of anomalous particle transport, the ratio between density and ion temperature gradient, $\eta_{i}$, can be expected to be found close to the value where the particle flux, generated by this mode, changes from outward to inward. This is consistent with experiments, where $\eta_{i}$ in the steep gradient zone is typically between 0.6 and 1.2 [35].

By another mechanism, this mode is also capable of setting the ratio between the electron temperature gradient and the density gradient. When starting at a very large value of $\eta_{e}$, there is a large amount of electron heat transport, which acts to reduce the temperature gradient. This reduces $\eta_{e}-$ which in turn reduces the turbulent electron heat transport - until a steady state is reached. On the other hand, for low $\eta_{e}$ values, when the mode is still linearly stable, the electron temperature gradient is allowed to grow (due to missing transport) until the mode becomes unstable again. Depending on the "stiffness factor" (i.e., on the slope of the flux-gradient relationship just above the linear threshold), which may be expected to be moderate for edge-ETG modes, the experimentally observed $\eta_{e}$ may then be expected to exceed the threshold value by a reasonable amount. The measured values in ASDEX Upgrade edge plasmas are in the range $\eta_{e} \sim 2$, i.e., about a factor of 1.6 above the threshold. Thus, by the mechanisms just explained, the edge-ETG mode may be able to determine the ratios of temperature and density gradients $\left(\eta_{i} \sim 1, \eta_{e} \sim 2\right)$ in the tokamak edge whenever it becomes the dominant channel of anomalous particle and electron heat flux.

The same kind of edge-ETG instability is present both in the L- and H-mode phases of shot \#22838. Here, we find that the growth rate increases as the experiment enters deeper into the $\mathrm{H}$-mode regime. This is due to the increase of $\eta_{e}$, which lies around 2.0 in a "deep H-mode" case, but only around 1.35 in our L-mode situation, the latter being very close to the critical value. It is reasonable to assume that this trend will carry over to nonlinear simulations, but on the other hand, the influence of $E \times B$ shear (which also increases when entering deeper into the $\mathrm{H}$-mode regime) on the edge-ETG mode has not yet been determined and may reverse this tendency.

\section{SUMMARY}

In this paper, we have presented comprehensive linear gyrokinetic simulations of ASDEX Upgrade edge plasmas based on realistic MHD equilibria. In this context, it turned out that the common list of microinstabilities (mentioned in the introduction) as the main agents of anomalous transport in tokamaks is incomplete.

We have examined three scenarios, ranging from an LMode (although close to the $\mathrm{L}-\mathrm{H}$ transition) to a deep $\mathrm{H}$-mode discharge. All over this rather broad range of experimental conditions, we found that the outer core region of these plasmas is susceptible to a microtearing instability which might enhance the electron heat flux in that region.

Furthermore, the steep gradients and highly shaped geometry in the plasma edge region lead to an ETG-like instability that exists down to very low wavenumbers and tends to peak near the X-point(s). It possesses properties which may explain the ratios of the density and temperature gradient scale lengths $\left(\eta_{e} \approx 2, \eta_{i} \approx 1\right)$ that have been consistently found in various experiments over the last decade.

To fortify the results we have shown, especially those regarding the transport properties in the steep gradientregion of tokamaks, nonlinear simulations will be essential, accompanied by further comparisons with experimentally measured heat and particle fluxes. In addition, the interplay between increasing $E \times B$ shear and increasing growth rates (when entering deeper into the $\mathrm{H}$-mode regime) needs to be assessed, as well as the influence of $E \times B$ shear on the larger-scale instabilities we found in the edge. These tasks are left for future work.

\section{Acknowledgements}

The authors wish to thank M. Kammerer, F. Merz, and T. Görler for valuable discussions and help with the GEnE code. The computations have been performed on the Bов Cluster at the Garching Computing Center. 
[1] E.J. Doyle, W.A. Houlberg, Y. Kamada, V. Mukhovatov, T.H. Osborne, A. Polevoi, G. Bateman, J.W. Connor, J.G. Cordey, T. Fujita, X. Garbet, T.S. Hahm, L.D. Horton, A.E. Hubbard, F. Imbeaux, F. Jenko, J.E. Kinsey, Y. Kishimoto, J. Li, T.C. Luce, Y. Martin, M. Ossipenko, V. Parail, A. Peeters, T.L. Rhodes, J.E. Rice, C.M. Roach, V. Rozhansky, F. Ryter, G. Saibene, R. Sartori, A.C.C. Sips, J.A. Snipes, M. Sugihara, E.J. Synakowski, H. Takenaga, T. Takizuka, K. Thomsen, M.R. Wade, H.R. Wilson, ITPA Transport Physics Topical Group, ITPA Confinement Database and Modelling Topical Group and ITPA Pedestal and Edge Topical Group, Nucl. Fusion 47, S18 (2007)

[2] O. Gruber, H.-S. Bosch, S. Günter, A. Herrmann, A. Kallenbach, M. Kaufmann, K. Krieger, K. Lackner, V. Mertens, R. Neu, F. Ryter, J. Schweinzer, A. Stäbler, W. Suttrop, R. Wolf, K. Asmussen, A. Bard, G. Becker, K. Behler, K. Behringer, A. Bergmann, M. BessenrodtWeberpals, K. Borrass, B. Braams, M. Brambilla, R. Brandenburg, F. Braun, H. Brinkschulte, R. Brückner, B. Brüsehaber, K. Büchl, A. Buhler, H.P. Callaghan, A. Carlson, D.P. Coster, L. Cupido, S. de Pea Hempel, C. Dorn, R. Drube, R. Dux, S. Egorov, W. Engelhardt, H.-U. Fahrbach, U. Fantz, H.-U. Feist, P. Franzen, J.C. Fuchs, G. Fussmann, J. Gafert, G. Gantenbein, O. Gehre, A. Geier, J. Gernhardt, E. Gubanka, A. Gude, G. Haas, K. Hallatschek, D. Hartmann, B. Heinemann, G. Herppich, W. Herrmann, F. Hofmeister, E. Holzhauer, D. Jacobi, M. Kakoulidis, N. Karakatsanis, O. Kardaun, A. Khutoretski, H. Kollotzek, S. Kötterl, W. Kraus, B. Kurzan, G. Kyriakakis, P.T. Lang, R.S. Lang, M. Laux, L.L. Lengyel, F. Leuterer, A. Lorenz, H. Maier, M. Manso, M. Maraschek, M. Markoulaki, K.-F. Mast, P.J. McCarthy, D. Meisel, H. Meister, R. Merkel, J.P. Meskat, H.W. Müller, M. Münich, H. Murmann, B. Napiontek, G. Neu, J. Neuhauser, M. Niethammer, J.-M. Noterdaeme, G. Pautasso, A.G. Peeters, G. Pereverzev, S. Pinches, G. Raupp, K. Reinmüller, R. Riedl, V. Rohde, H. Röhr, J. Roth, H. Salzmann, W. Sandmann, H.B. Schilling, D. Schlögl, K. Schmidtmann, H. Schneider, R. Schneider, W. Schneider, G. Schramm, S. Schweizer, R.R. Schwörer, B.D. Scott, U. Seidel, F. Serra, S. Sesnic, C. Sihler, A. Silva, E. Speth, K.-H. Steuer, J. Stober, B. Streibl, A. Thoma, W. Treutterer, M. Troppmann, N. Tsois, W. Ullrich, M. Ulrich, P. Varela, H. Verbeek, O. Vollmer, H. Wedler, M. Weinlich, U. Wenzel, F. Wesner, R. Wunderlich, N. Xantopoulos, Q. Yu, D. Zasche, T. Zehetbauer, H.-P. Zehrfeld, H. Zohm and M. Zouhar Nucl. Fusion 39, 1321 (1999)

[3] P.C. Liewer, Nucl. Fusion 25, 543 (1985)

[4] A. Hasegawa and K. Mima, Phys. Rev. Lett. 39, 205 (1977)

[5] A. Zeiler, D. Biskamp, J.F. Drake, and P.N. Guzdar, Phys. Plasmas 3, 2951 (1996)

[6] A. Zeiler, D. Biskamp, J.F. Drake, and B.N. Rogers, Phys. Plasmas 5, 2654 (1998)

[7] B.N. Rogers, J.F. Drake, and A. Zeiler, Phys. Rev. Lett. 81, 4396 (1998)

[8] J.W. Connor, R.J. Hastie, and J.B. Taylor, Phys. Rev. Lett. 40, 396 (1978)

[9] B. Scott, New J. Phys. 4, 52 (2002)
[10] B. Scott, Phys. Plasmas 7, 1845 (2000)

[11] B. Scott, Phys. Plasmas 8, 447 (2001)

[12] F. Jenko, J. Plasma Fusion Res. SERIES 6, 11 (2004)

[13] F. Jenko, W. Dorland, M. Kotschenreuther, and B.N. Rogers, Phys. Plasmas 7, 1904 (2000)

[14] T. Dannert and F. Jenko, Phys. Plasmas 12, 072309 (2005)

[15] X.Q. Xu und M.N. Rosenbluth, Phys. Fluids B 3, 627 (1991)

[16] F. Merz, Doctoral thesis, University of Münster (2008).

[17] P. Xanthopoulos and F. Jenko, Phys. Plasmas 13, 092301 (2006)

[18] R. J. Groebner and T. H. Osborne, Phys. Plasmas 5, $1800(1998)$

[19] T. Puetterich, C.F. Maggi, L.D. Horton, R. Dux, B. Langer, E. Wolfrum, and the ASDEX Upgrade Team, Proceedings of Contributed Papers, 35th European Physical Society Conference on Plasma Physics, Hersonissos, 2008, P2.083

[20] R.E. Waltz, J. Candy, and M. Fahey, Phys. Plasmas 14, $056116(2007)$

[21] T. Görler and F. Jenko, Phys. Rev. Lett. 100, 185002 (2008)

[22] F. Jenko, W. Dorland, and G.W. Hammett, Phys. Plasmas 8, 4096 (2001)

[23] L. Vermare, C. Angioni, A. Bottino, A.G. Peeters and the ASDEX Upgrade Team, J. Phys.: Conf. Ser. 123, 012040 (2008)

[24] D.J. Applegate, C.M. Roach, J.W. Connor, S.C. Cowley, W. Dorland, R.J. Hastie and N. Joiner, Plasma Phys. Control. Fusion 49, 1113 (2007)

[25] R.D. Hazeltine, D. Dobrott, and T.S. Wang, Phys. Fluids 18, 1778 (1975)

[26] K.L. Wong, S. Kaye, D.R. Mikkelsen, J.A. Krommes, K. Hill, R. Bell, and B. LeBlanc, Phys. Rev. Lett. 99, 135003 (2007)

[27] C.M. Roach, D.J. Applegate, J.W. Connor, S.C. Cowley, W.D. Dorland, R.J. Hastie, N. Joiner, S. Saarelma, A.A. Schekochihin, R.J. Akers, C. Brickley, A.R. Field, M. Valovic and the MAST Team, Plasma Phys. Control. Fusion 47, B323 (2005)

[28] J.F. Drake and Y.C. Lee, Phys. Fluids 20, 1341 (1977)

[29] J.W. Connor, S.C. Cowley, and R.J. Hastie, Plasma Phys. Control. Fusion 32, 799 (1990)

[30] B. Coppi, F. Pegoraro, Nucl. Fusion 17, 969 (1977)

[31] J.W. Connor, C.M. Roach, R.J. Hastie, P. Helander, T.J. Martin, D.J. Applegate, N. Joiner, M. Reshko, S. Saarelma, W.D. Dorland, S.C. Cowley, S. Newton and A.R. Field for the MAST Team, Fusion Energy 2006, Proceedings of 21st International Conference, Chengdu, IAEA, TH/P2-2, (2006)

[32] M. Kammerer, F. Merz, and F. Jenko, Phys. Plasmas 15, $052102(2008)$

[33] J.F. Drake, P.N. Guzdar, and A.B. Hassam, Phys. Rev. Lett. 61, 2205 (1988)

[34] F. Jenko, W. Dorland, A. Kendl, and D. Strintzi, Simulation and Theory of Electron Temperature Gradient Turbulence, in Theory of Fusion Plasmas (SIF, Bologna, 2002)

[35] E. Wolfrum, D. Coster, C. Konz, M. Reich and the ASDEX Upgrade Team, Proceedings of Contributed Papers, 
34th European Physical Society Conference on Plasma

Physics, Warsaw, 2007, Vol. 31F, P2.039 